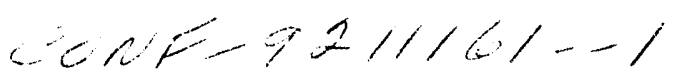

The submitted menuscript hes been whored by a contractor of the U.S. Government under contrect Na. W.31.YOOENG-3a. Accordingly. the U.S Gowmment reteins nonexctusive, rovelty-trie licanse to publinh or reproduce the publimed form of thin C. S. Gowion, or wllow others to to to for
U. form.

\title{
The Canonical Forms of a Lattice Rule
}

\author{
J. N. Lyness
}

ANL/MCS/CP-78196

DE93 005686

\begin{abstract}
Much of the elementary theory of lattice rules may, be presented as an elegant application of classical results. These include Kronecker group representation theorem and the Hermite and Smith normal forms of integer matrices. The theory of the canonical form is a case in point. In this paper, some of this theory is treated in a constructive rather than abstract manner. A step-by-step approach that parallels the group theory is described, leading to an algorithm to obtain a canonical form of a rule of prime power order. The number of possible distinct canonical forms is derived, and this is used to determine the number of integration lattices having specified invariants.
\end{abstract}

\section{Notation Used to Describe and Classify Lattice Rules}

An s-dimensional lattice, $\Lambda$, is a set of points having the property that, when $\mathbf{p}$ and $q$ are members of $\Lambda$, so are $p+q$ and $p-q$. It may be defined by this property, together with a restriction that there are no points of accumulation. A very familiar lattice is the unit lattice $\Lambda_{0}$, which comprises all points $\mathbf{p}=\left(p_{1}, p_{2}, \ldots, p_{s}\right)$, all of whose components $p_{i}$ are integers. An integration lattice is a lattice that contains the unit lattice $I_{0}$ as a sublattice. A lattice rule $Q(\Lambda)$ is a quadrature rule for $[0,1)^{\circ}$ that employs the points of $\Lambda \cap[0,1)^{s}$ as an abscissa set $A(Q)$ and assigns an equal weight to each. Some lattice rules are useful for integrating naturally periodic functions. Other lattice rules may be very inefficient. A representative selection of the literature ou lattice rules may be found in Sloan (1992) and Niederreiter (1992).

The investigation of lattice rules is hampered by two features: first, the large numbers of different rules that are available, and second, a bewildering lack of uniqueness in the various convenient representations for investigating rules and for classifying them.

A classical approach to lattices is based on the generator matrix. It is readily shown that, given any $s$-dimensional lattice $\Lambda$, there exists a set of $s$ generators $a_{1}, a_{2}, \ldots, a_{2}$ such that all points

$$
\mathbf{p}=\sum_{i=1}^{\infty} \lambda_{i} a_{i} \quad \lambda_{i} \text { integer }
$$

lie in the lati ie, and all lattice points are of this form. The $s \times s$ matrix $A$ whose $j$-th row is $\mathbf{a}_{j}(j=1,2, \ldots, s)$ is referred to as a generator matrix of the lattice. When 
$\Lambda$ is an integration lattice, it is readily shown that all elements of $A$ are rational and that $N=|\operatorname{det} A|^{-1}$ is the order of $A(Q)$, the abscissa set of $Q(\Lambda)$. An approach based particularly on the generator matrix $\left(A^{-1}\right)^{T}$ of the reciprocal lattice $\Lambda^{\perp}$ has proved fruitful. However, inconvenient aspects of this approach include the absence of uniqueness of $A$ and the difficulty of proceeding from the matrix $A$ to a sum of function values.

The standard number theoretic rules of Korobov (1959) are also iattice rules. These are conventionally expressed in the form

$$
Q f=\frac{1}{n} \sum_{j=1}^{n} \bar{f}\left(\frac{\mathbf{z}}{n} j\right) .
$$

Here $\mathbf{z} \in \Lambda_{0}$ and $\bar{f}(\mathbf{x})$ is a periodic continuation of $f(\mathbf{x})$ which coincides with $f(\mathbf{x})$ in $[0,1)^{s}$. As is conventional, we denote $\mathbf{x}$ modulo $\Lambda_{0}$ by $\{\mathbf{x}\}$. The components of $\{\mathbf{x}\}$ are the respective nonnegative fractional parts of the components of $\mathbf{x}$.

Only some lattice rules (those of rank 1) can be expressed in this form. On the other hand, all lattice rules may be expressed in a natural generalization of form (1.1) that we refer to as a $t$-cycle $D-Z$ rule form. This is

$$
Q f=\frac{1}{d_{1} d_{2} \ldots d_{t}} \sum_{j_{1}=1}^{d_{1}} \sum_{j_{2}=1}^{d_{2}} \cdots \sum_{j_{t}=1}^{d_{t}} \bar{f}\left(\sum_{i=1}^{t} \frac{z_{i}}{d_{i}} j_{i}\right),
$$

where $d_{i}$ are integers and $\mathbf{z}_{i} \in \Lambda_{0}$. Associated with this form are two integer matrices. $D=\operatorname{diag}\left\{d_{1}, d_{2}, \ldots, d_{t}\right\}$ is a $t \times t$ diagonal matrix, and $Z$ is the $t \times s$ matrix whose $j$-th row is $\mathbf{z}_{j}$.

It is not difficult to show that this form represents a lattice rule. In fact, it is the rule of lowest order $N$ that includes

$$
\mathbf{c}_{i}=\left\{\mathbf{z}_{i} / d_{i}\right\} \quad i=1,2, \ldots, t .
$$

The lattice $\Lambda$ is generated by these $\mathbf{c}_{i}$ together with the unit vectors $\mathbf{e}_{k}, k=1,2, \ldots, s$. Note that the $m$-panel product trapezoidal rule is a lattice rule. One of its $s$-cycle $D-Z$ forms has $D=m I$ and $Z=I$.

Form (1.2) is not unique. Moreover, it may be repetitive. That is, for some integer $k$, it may include only $d_{1} d_{2} \ldots d_{t} / k$ distinct abscissas, each repeated $k$ times. This may happen trivially.

Definition 1.2 The component $\mathbf{z} / n$, where $\mathbf{z}=\left(\zeta_{1}, \zeta_{2}, \ldots, \zeta_{s}\right) \in \Lambda_{0}$ is termed proper when $\left(\zeta_{1}, \zeta_{2}, \ldots, \zeta_{s}, n\right)=1$ and $\mathbf{c}=\mathbf{z} / n \in[0,1)^{s}$. (Colloquially, $\mathbf{z} / n$ is in its lowest terms.)

If $\mathbf{z} / n$ in (1.1) is not proper, then the form is repetitive. This sort of trivial repetition is easy to recognize. However, possible repetition in the general $t$-cycle $D-Z$ form need not be at all obvious. 
It is straightforward to show (see Sloan and Lyness 1989) that a necessary and sufficient condition for the rule form $Q f$ in (1.2) to be repetitive is that there exist integers $j_{1}, j_{2}, \ldots, j_{t}$ with $j_{i} \in\left[0, d_{i}\right)$, not all zero, such that

$$
\sum_{i=1}^{t} \frac{j_{i} \mathbf{z}_{i}}{d_{i}}=\text { integer. }
$$

One classification of lattice rules based on the $t$-cycle form relies on the circumstance that the elements of the abscissa set $A(Q)$ form a group $G$ under addition modulo $\Lambda_{0}$.

In Sloan and Lyness (1989), the Kronecker group representation theorem was applied to this group to show that a $t$-cycle nonrepetitive $D-Z$ form exists in which all elements $d_{i}$ exceed 1 , and $d_{i} \mid d_{i-1}$, and the $z_{i}$ are linearly independent. Such a rule form was termed a canonical form. From the nomenclature of group theory, these particular values of $d_{i}$ are termed invariants, and this particular value of $t$ is termed the rank of the rule $Q$. The rank and invariants are unique to the lattice, but the choice of $\mathbf{z}_{i}$ in the canonical form is far from unique. However, no constructive approach for proceeding from a general $t$-cycle form to a canonical form was made available at that time.

This paper is concerned with finding a canonical form of a rule defined by a general $t$-cycle $D-Z$ form. We shall, in fact, show only how to find nonrepetitive forms in which $d_{i+1} \mid d_{i}$. We may then rely on Corollary 3.6 of Sloan and Lyness (1989) which assures us that a form that appears to be canonical is indeed canonical if it is nonrepetitive.

\section{Decompösition and Reassembly}

The accompanying table illustrates the underlying group theory.

\begin{tabular}{l|llll}
$G$ & $F_{1}$ & $F_{2}$ & & $F_{s}$ \\
\hline$S_{1}^{\prime}$ & $E_{11}$ & $E_{12}$ & $\ldots$ & $E_{1 s}$ \\
$S_{2}^{\prime}$ & $E_{21}$ & $E_{22}$ & $\ldots$ & $E_{2 s}$ \\
$\vdots$ & & & & \\
$S_{q}^{\prime}$ & $E_{q 1}$ & $E_{q 2}$ & $\ldots$ & $E_{q s}$ \\
\hline
\end{tabular}

Each element is an abelian group. Any group in the initial row (column) is the direct sum of the other groups in that row (column), and the groups in all but the initial column are cyclic groups. The table illustrates the decomposition of an abelian group $G$ of order $N=p_{1}^{\beta_{1}} p_{2}^{\beta_{2}} \ldots p_{q}^{\beta_{q}}$ into the direct sum of cyclic groups $F_{k}$. Here 
$p_{1}, p_{2}, \ldots, p_{q}$ are distinct primes, and $\beta_{i}$ are positive integers. The first stage is the decomposition of $G$ into the direct sum

$$
G=S_{1} \oplus S_{2} \oplus \ldots \oplus S_{q}
$$

of its Sylow $p$ groups. $S_{j}$, which is of order $p_{j}^{\beta_{j}}$, contains all elements of $G$ whose order is any integer power of $p_{j}$. Any noncyclic abelian group of prime power order may be decomposed into a direct sum of cyclic groups. Thus,

$$
S_{j}=E_{j, 1} \oplus E_{j, 2} \oplus \ldots \oplus E_{j, s}
$$

where $E_{j, k}$ is a cyclic group of order $p_{j}^{\beta_{, k}}$. These have been arranged in order so that $\beta_{j, k} \geq \beta_{j, k+1}$. Clearly, $\sum_{k=1}^{s} \beta_{j, k}=\beta_{j}$. There may be fewer nontrivial groups than indicated here. The theory is not compromised if some trivial groups $E_{j, k}$ are included but ignored. For these, $\beta_{j, k}=0$.

Finally, we apply the result that the direct sum of cyclic groups whose orders are mutually prime is also a cyclic group. Thus,

$$
F_{k}=\dot{E}_{1, k} \oplus E_{2, k} \oplus \ldots \oplus E_{s, k}
$$

is a cyclic group of order $n_{k}=\prod_{j=1}^{q} p_{j}^{\beta_{j k}}$. Note that, since $\beta_{j, k} \geq \beta_{j, k+1}$, it follows that $n_{k+1} \mid n_{k}$.

What is illustrated here is the Kronecker decomposition of an abelian group $G$ into the direct sum of $s$ cyclic groups $F_{k} ; k=1,2, \ldots, s$. The nontrivial values of $n_{k}$ are termed the invariants of $G$, and the number of these is termed the rank of $G$.

The above remarks comprise at most a schematic for a possible derivation of a far. ous theorem in group theory. For our purposes, as we shall see, we do not need group theory. We may use the schematic to derive a canonical form.

It appears that in our application, the decomposition (2.1) into Sylow $p$ groups is very simple, as is the recomposition (2.3) of the cyclic groups $E_{j, k}$ into $F_{k}$. However, the middle stage (2.2) is nontrivial. The following examples illustrate these outer operations. These are justified in the following theorem.

Theorem 2.4 Let $n=p q,(p, q)=1$, and $\mathbf{z}, \mathbf{z}_{1}, \mathbf{z}_{2} \in \Lambda_{0}$. Then

$$
\sum_{j=1}^{n} \bar{f}\left(\frac{j \mathbf{z}}{n}+\epsilon\right)=\sum_{j_{1}=1}^{p} \sum_{j_{2}=1}^{q} \bar{f}\left(\frac{j_{1} \mathbf{z}}{p}+\frac{j_{2} \mathbf{z}}{q}+\epsilon\right)
$$

and

$$
\sum_{j_{1}=1}^{p} \sum_{j_{2}=1}^{q} \bar{f}\left(\frac{j_{1} \mathbf{z}_{1}}{p}+\frac{j_{2} \mathbf{z}_{2}}{q}+\epsilon\right)=\sum_{j=1}^{n} \bar{f}\left(\frac{j \mathbf{z}_{3}}{p q}+\epsilon\right),
$$

where $\mathbf{z}_{3}=q \mathbf{z}_{1}+p \mathbf{z}_{2}$. Moreover, if $\mathbf{z}_{1} / p$ and $\mathbf{z}_{2} / q$ are proper (see (1.3) above), so is $\left\{\mathbf{z}_{3} / p q\right\}$. 
The proof of either part is trivial. Note that there are no minor restrictions. For example, $z_{2} / q$ need not be in its lowest terms. The $\epsilon$ is notationally helpful in establishing extensions of these results.

As an example, we put the following rule into canonical form.

\section{Example 2.5}

$$
Q f=\frac{1}{12.15} \sum_{j_{1}=1}^{12} \sum_{j_{2}=1}^{15} \bar{f}\left(\frac{(5,8,3)}{12} j_{1}+\frac{(8,1,0)}{15} j_{2}\right) .
$$

This may be reexpressed as

$$
\frac{1}{4.3 .3 .5} \sum_{j_{1}=1}^{4} \sum_{j_{2}=1}^{3} \sum_{j_{3}=1}^{3} \sum_{j_{4}=1}^{5} \bar{f}(\mathbf{x})
$$

where

$$
\mathbf{x}=\frac{(5,8,3)}{4} j_{1}+\frac{(5,8,3)}{3} j_{2}+\frac{(8,1,0)}{3} j_{3}+\frac{(8,1,0)}{5} j_{4}
$$

This may be replaced by

$$
\mathbf{x}^{\prime}=\frac{(1,0,3)}{4} j_{1}+\frac{(2,2,0)}{3} j_{2}+\frac{(2,1,0)}{3} j_{3}+\frac{(3,1,0)}{5} j_{4}
$$

The sum in (2.5), which contains 180 elements of $G$, has now been reexpressed as the direct sum of three Sylow $p$ groups, with $p=2,3$, and 5 , respectively. Those with $p=2$ and 5 are already cyclic groups. The Sylow 3 group is the direct sum of two cyclic groups:

The intermediate stage comprises, in general, removing repetition from each of the Sylow $p$ groups. In this case, it is clear by inspection that the groups of order 3 are distinct. That is, the sums over $j_{2}$ and $j_{3}$ include nine distinct terms and not three. Applying the second part of Theorem 2.4 twice, we find successively

$$
\begin{aligned}
Q f & =\frac{1}{180} \sum_{j_{12}} \sum_{j_{3}} \sum_{j_{4}} \bar{f}\left(\mathbf{x}^{\prime \prime}\right) \\
& =\frac{1}{180} \sum_{j_{124}} \sum_{j_{4}} \bar{f}\left(\mathbf{x}^{\prime \prime \prime}\right) \\
& =\frac{1}{180} \sum_{k_{1}=1}^{60} \sum_{k_{2}=1}^{3} \bar{f}\left(\frac{(31,52,45)}{60} k_{1}+\frac{(2,1,0)}{3} k_{2}\right) .
\end{aligned}
$$

Here

$$
\mathbf{x}^{\prime \prime}=\frac{(11,8,9)}{12} j_{12}+\frac{(2,1,0)}{3} j_{3}+\frac{(3,1,0)}{5}
$$

and

$$
\mathbf{x}^{\prime \prime \prime}=\frac{(91,52,45)}{60} j_{124}+\frac{(2,1,0)}{3} j_{3}
$$


This rule form (2.6) is in canonical form.

The reader may have noticed that the overall result can be significantly altered by what appears at first sight to be a minor change in the problem. For example, if in Example 2.5 we replace the second vector $(8,1,0)$ by $(7,1,0)$, we find that the vector $(2,1,0)$ in $\mathbf{x}^{\prime}$ must be replaced by $(1,1,0)$. The middle stage becomes

$$
\sum_{j_{2}=1}^{3} \sum_{j_{3}=1}^{3} \bar{f}\left(\frac{(2,2,0)}{3} j_{2}+\frac{(1,1,0)}{3} j_{3}+\epsilon\right)=3 \sum_{j_{23}=1}^{3} \bar{f}\left(\frac{(1,1,0)}{3} j_{23}+\epsilon\right) .
$$

The new form $Q f$ is repetitive, and $Q$ has one invariant instead of two.

A satisfactory procedure to obtain the canonical form cannot rely on the chance recognition of this sort of circumstance. Subsequent sections are devoted to providing an algorithm for handling this problem.

\section{Rules of Prime Power Order}

In this section we deal with lattices and rule forms of prime power order; that is, a $t$ cycle $D-Z$ rule form in which

$$
d_{j}=p^{\alpha,} \quad j=1,2, \ldots, t
$$

where $p$ is a prime. We are concerned with recognizing that this form is not repetitive or, if it is, reducing it to a new form that is not repetitive.

As a preliminary, we remove some trivial complications. We are interested only in sequential proper forms. $Q f$ is sequential if $d_{1} \geq d_{2} \geq \ldots \geq d_{t}>1$. The $i$-th component is proper if $\mathbf{z}_{i} / d_{i}$ is in its lowest terms and $\mathbf{c}_{i}=\mathbf{z}_{i} / d_{i} \in[0,1)^{s}$. The form is sequential proper when it is sequential and each element is proper. It is a trivial task to reexpress $Q f$ in a $t^{\prime}$ cycle $D-Z$, sequential proper form.

A geometric view is that the rule form is defined by $r$ points $\mathbf{c}_{1}, \mathbf{c}_{2}, \ldots, \mathbf{c}_{t}$ of orders $d_{1}, d_{2}, \ldots ; d_{t}$, respectively. It is in sequential proper form when $\mathbf{c}_{i} \in[0,1)^{s}$ and $d_{1} \geq d_{2} \ldots \geq d_{t}>1$. It still may be repetitive (trivially if $\mathbf{c}_{i}=\mathbf{c}_{i+1}$ and $d_{i}=d_{i+1}$ ).

Theorem 3.2 Let $Q f$ and $Q^{\prime} f$ be t-cycle $D-Z$ forms having the same $Z$ matrix. Let $D=\operatorname{diag}\left(d_{1}, d_{2}, \ldots, d_{t}\right)$ and $D^{\prime}=\operatorname{diag}(p, p, \ldots, p)=p I$. Then $Q f$ and $Q^{\prime} f$ are either both repetitive or both nonrepetitive.

Proof. The condition for $Q f$ to be repetitive is that there exist integers $j_{i} \in\left[0, d_{i}\right)$ $i=1,2, \ldots, t$ not all zero such that

$$
\sum_{i=1}^{t} j_{i} \mathbf{z}_{i} / d_{i}=\text { integer. }
$$


Let us suppose that $Q f$ is repetitive and the above $j_{i}$ exist. Let $I^{\prime}$ be $\left\{i \mid j_{i} \neq 0\right\}$. Let $j_{i} / d_{i}=\gamma_{i} / m_{i}$ in their lowest terms. Then $\gamma_{i} \neq 0, i \in I^{\prime}$, and

$$
\sum_{i \in I^{\prime}} \gamma_{i} \mathbf{z}_{i} / m_{i}=\text { integer. }
$$

Let $p^{\lambda}=\max _{i \in I^{\prime}} m_{i}$. Let $I^{\prime \prime}$ be $\left\{i \mid m_{i}=p^{\lambda}\right\}$. Then multiplying (3.4) by $p^{\lambda-1}$ and subsuming all integers to the right gives

$$
\sum_{i \in I^{\prime \prime}} \gamma_{i} \mathbf{z}_{i} / p=\text { integer. }
$$

This statement implies that $Q^{\prime} f$ is repetitive.

The converse is marginally simpler to prove. If we set

$$
\begin{array}{ll}
j_{i}=\gamma_{i} d_{i} / p & i \in I^{\prime \prime} \\
j_{i}=0 & i \notin I^{\prime \prime},
\end{array}
$$

we recover (3.3) from (3.5).

Recasting this theorem geometrically is revealing.

Corollary 3.6 Any nonrepetitive prime power $t$-cycle rule form includes precisely $p^{t}-1$ distinct points of order $p$.

The theorem shows that the critical quantities in determining whether a prime power form is repetitive is the $Z$ matrix. The integers $d_{i}$ play a secondary role. This is confirmed in the following theorem.

Theorem 3.7 A t-cycle $D-Z$ form of a p-power rule is repetitive if and only if the rank of $Z$ modulo $p$ is less than $t$.

Proof: In view of Theorem 3.2, we need only establish this for a $t$-cycle $D^{\prime}-Z$ form where

$$
D^{\prime}=\operatorname{diag}\{p, p, \ldots, p\}
$$

If this is repetitive, there exist $j_{1}, j_{2}, \ldots, j_{t}$, not all zero such that

$$
\left\{\sum_{i=1}^{t} j_{i} \mathbf{z}_{i} / p\right\}=0 .
$$

This implies that $\sum_{i=1}^{t} j_{i} \mathbf{z}_{i}=p \mathbf{u}$ for some $\mathbf{u} \in \Lambda_{0}$; this is the condition that the rank of $Z$ modulo $p$ is less than $t$.

The simple results of this section may be readily incorporated into an algorithm that transforms a general $p$-power $t$-cycle $D-Z$ rule form into a sequential, proper, and 
scaled canonical form. In the final form, $Z$ either is unimodular or contains a $t \times t$ unimodular submatrix.

This algorithm is a variant of a standard triangularization technique.

At the start of the $j$-th stage, we have

$$
d_{1} \geq d_{2} \geq \ldots \geq d_{j} \geq \ldots \geq d_{t}>1
$$

all being powers of $p$ and $\mathbf{z}_{i} / d_{i} i=1 \ldots t$ are all proper. Associated with $\mathbf{z}_{1}, \mathbf{z}_{2}, \ldots, \mathbf{z}_{j-1}$ we have distinct integers $i_{1}, i_{2}, \ldots, i_{j-1}$, which we term column indices. Moreover,

$$
\left.\begin{array}{ll}
Z_{r, i_{c}}=1 & \text { when } \quad r=c \\
Z_{r, i_{c}}=0 & \text { when } \quad r>c
\end{array}\right\} \begin{aligned}
& c=1,2, \ldots, j-1 \\
& r=1,2, \ldots, t
\end{aligned}
$$

The $j$-th stage comprise" the following:

1. Let $\mathbf{z}_{j}=\left(\zeta_{1}, \zeta_{2}, \ldots, \zeta_{s}\right)$. Identify a component, say $\zeta_{\ell}$, for which $\left(\zeta_{\ell}, p\right)=1$. Set $i_{j}=\ell$. Replace $\mathbf{z}_{j}$ by $k \mathbf{z}_{j}$, where $k=\zeta_{\ell}^{-1}\left(\bmod d_{j}\right)$ and then replace $\mathbf{z}_{j}$ by $\mathbf{z}_{j}^{\prime}=\left(\zeta_{1}^{\prime}, \zeta_{2}^{\prime}, \ldots, \zeta_{\ell}^{\prime}\right)$ with $\zeta_{i}^{\prime} \in\left[0, d_{j}\right)$. (This leaves $\zeta_{\ell}^{\prime}=1$.)

2. For $i>j$, replace $\mathbf{z}_{i}$ by $\mathbf{z}_{i}-Z_{i, \ell} \mathbf{z}_{j}$. (This leaves $Z_{i, \ell}=0 ; i>j$.)

3. Carry out trivial adjustments on $Z$ and $D$ necessary to leave a $t^{\prime}$-cycle $D-Z$ sequential proper form.

\section{Comments:}

1. We have by hypothesis that $\mathbf{z}_{j} / d_{j}$ is proper. Thus, there is some component of $z_{j}$ that is not a multiple of $p$, and that value of $j$ can be chosen for $i_{j}$ in 1 .

3. These adjustments include:
a. Put ăny improper $\mathbf{z}_{i} / d_{i}$ into proper form $\mathbf{z}_{i}^{\prime} / d_{i}^{\prime}$.
b. Remove any rows $\mathbf{z}_{i}$ for which either $\mathbf{z}_{i}=\mathbf{0}$ or $d_{i}=1$; naturally this step reduces the value of $t$.
c. If necessary, reorder the rows of $Z$ so that the ordering of $d_{i}$ is sequential.

The final $Z$ matrix is an integer matrix. If its columns were permuted in accordance with the column indices, it would be upper triangular with unit diagonal. Thus, it contains a $t \times t$ unimodular submatrix (corresponding to retaining only the columns numbered $i_{1}, i_{2}, \ldots, i_{t}$ ).

Because of this unimodularity, it follows that when $t=s$, the matrix $A=D^{-1} Z$ is a generator matrix of $\Lambda$. In general, $t<s$ and $\tilde{A}=D^{-1} Z$ comprises the first $t$ rows of a 
generator matrix. The remaining $s-t$ rows of the generator matrix may be chosen as unit vectors $\mathbf{e}_{\lambda}$, the $\lambda$ being the elements of $[1, s]$ not assigned to be column indices.

Finally, note that $Z$ is not unique. In general, the column indices can be chosen in many ways, and rows having the same $d$ element may be interchanged.

\section{Canonical Form Redundancy of Prime Power Forms}

In the preceding section, we remarked that the $Z$ matrix is not unique. In this section, we quantify this lack of uniqueness. We take an $r$-cycle prime power rule form $Q f$ which is in canonical form; we see how many distinct reassignments of $Z$ exist. Initially, we shall have to assume that the result depends on $p, D$, and $Z$. However, it will appear that it simpiy depends on $p$ and $D$. Let

$$
Q f=\frac{1}{n_{1} n_{2} \ldots n_{r}} \sum_{j_{1}=1}^{n_{1}} \sum_{j_{2}=1}^{n_{2}} \ldots \sum_{j_{r}=1}^{n_{r}} \bar{f}\left(\sum_{k=1}^{r} \frac{j_{k} \mathbf{z}_{k}}{n_{k}}\right)
$$

be a canonical form of $Q$. Here as before,

$$
n_{1} \geq n_{2} \geq \ldots \geq n_{r}>1
$$

and each $n_{i}$ is a power of $p$. Also, $\mathbf{z}_{k} \in\left[0, n_{k}\right), k=1,2, \ldots, r$. Note that, since this is a canonical form, we know that $\mathbf{z}_{k} / n_{k}$ is in its lowest terms. Each member of the set of generators

$$
\mathbf{c}_{k}=\mathbf{z}_{k} / n_{k}
$$

is itself an element of $[0,1)^{s}$.

Definition $4.3 \overline{\bar{\mu}}_{s}\left(n_{1}, n_{2}, \ldots, n_{s} ; N ; Z\right)$ is the number of distinct ways of assigning the $Z$-matrix so that the rule is the same. (Here $\mathbf{z}_{k} \in\left[0, n_{k}\right)$.)

Geometrically, this is the number of different point sets $\mathbf{c}_{1}, \mathbf{c}_{2}, \ldots, \mathbf{c}_{r}$ that can be put into (4.1) leaving the same rule. These proints are $\in[0,1)^{s}$.

It will appear that $\overline{\bar{\mu}}_{s}$ is inderendent of $Z$, and we shall later drop the $Z$ in the definition.

The construction of a formula for $\overline{\bar{\mu}}_{s}$ uses relatively straightforward concepts that nevertheless must be applied with care. We shall suppose that we assign the vectors $\mathbf{z}_{j}$ (or $\mathbf{c}_{j}=\mathbf{z}_{j} / n_{j}$ ) in turn, starting with $j=1$ and ending with $j=r$. Clearly, every $\mathbf{c}_{j}$ has to be a member of the abscissa set. Thus, when we start the $j$-th stage, we may limit the choice for $\mathbf{c}_{j}^{\prime}$ as follows:

$$
\mathbf{c}_{j}^{\prime}=\sum_{i=1}^{r} \lambda_{i}^{j} \mathbf{c}_{i} \quad \lambda_{i}^{j} \in\left[0, n_{i}\right)
$$

or

$$
\mathbf{z}_{j}^{\prime}=\sum_{i=1}^{r} \lambda_{i}^{j} \frac{n_{j}}{n_{i}} \mathbf{z}_{i}
$$


It is convenient to drop the \{\} symbol here. The restriction $\lambda_{i}^{j} \in\left[0, n_{i}\right)$ in $(4.4)$ simply prevents duplication, since $\lambda_{i}^{j} \mathbf{c}_{i} \equiv\left(\lambda_{i}^{j}+n_{i}\right) \mathbf{c}_{i}$. When assigning $\mathbf{c}_{j}^{\prime}$, it is clear that for those values of $i$ for which $n_{i}<n_{j}$, we may choose any available $\lambda_{i}^{j}$, and hence there are $n_{i}$ distinct choices for each such $\lambda_{i}^{j}$. However, for those values of $i$ for which $n_{i}>n_{j}$, the choice has to be restricted so that $\mathbf{z}_{j}^{\prime}$ in (4.5) turns out to be in $\Lambda_{0}$. That is, $\lambda_{i}^{j}\left(n_{j} / n_{i}\right)$ must be an integer. Thus, for these values of $i$, there are only $n_{j}$ distinct choices for each $\lambda_{i}^{j}$. (Geometrically, this recognizes that $\mathbf{c}_{j}$ is of order $n_{j}$ and therefore only multiples of $\mathbf{c}_{i}$ of this order or less may be included.) There remain values of $i$ for which $n_{i}=n_{j}$.

To fix ideas, suppose $n_{j+1}>n_{j}>n_{j-1}$. Then there is only one value $\lambda_{j}^{j}$ remaining to assign. Since $\mathbf{z}_{j}$ itself must be attainable, we can allow $\lambda_{j}^{j}$ to take one of $n_{j}\left(1-\frac{1}{p}\right)$ distinct values only.

In this case, then, the number of ways of assigning $\mathbf{z}_{j}^{\prime}$ is the product over $i$ of the number of ways of assigning $\lambda_{j}^{i}$. This gives

$$
\prod_{i=1}^{j-1} n_{j} \times n_{j}\left(1-\frac{1}{p}\right) \times \prod_{i=j+1}^{r} n_{i} .
$$

Thus, when $n_{j-1}=n_{j}>n_{j+1}$, whatever the individual values of $\mathbf{z}_{i}, i=1,2, \ldots, r$, the number of ways of reassigning $z_{j}$, without changing any other $z_{i}$ is

$$
n_{j}^{j} \prod_{i=j+1}^{r} n_{r}\left(1-\frac{1}{p^{\lambda(j)}}\right) \text { with } \lambda(j)=1 .
$$

In general, we cannot expect all $n_{j}$ to be distinct. Let us suppose

$$
n_{j}>n_{j+1}=n_{j+2}=\ldots=n_{j+w}>n_{j+w+1}
$$

and isolate the part of the calculation involving the assignment of $\mathbf{c}_{j+1}^{\prime} \ldots \mathbf{c}_{j+w}^{\prime}$ to the extent that this is affected by $\mathbf{c}_{j+1} \ldots \mathbf{c}_{j+w}$. For convenience, we suppress the subscript $j$ and denote by $n$ the common value $n_{j+1}$. We need to consider the number of possibilities for

$$
\begin{aligned}
& \mathbf{c}_{1}^{\prime}=\sum_{i=1}^{w} \lambda_{i}^{1} \mathbf{c}_{i} \\
& \mathbf{c}_{2}^{\prime}=\sum_{i=1}^{w} \lambda_{i}^{2} \mathbf{c}_{i} \\
& \mathbf{c}_{u}^{\prime}=\sum_{i=1}^{w} \lambda_{i}^{w} \mathbf{c}_{i} .
\end{aligned}
$$

These are assigned in the following order. First, $\lambda_{\imath}^{1}, i=1,2, \ldots w$ are assigned. We need $\mathbf{c}_{1}^{\prime}$ to be of order $n$. To ensure this, $\lambda_{i}^{1}$ may be chosen in any way so long as at least one term $\lambda_{i}^{1} \mathbf{c}_{i}$ is of order $n$. This is equivalent to choosing a point of order $n$ in a $w$-dimensional space. The number of points is $n^{w}\left(1-p^{-w}\right)$. Later in the calculation, 
we have to assign $\mathbf{c}_{2}^{\prime}$. However, $\mathbf{c}_{1}$ has already been assigned and is of order $n$. To ensure that $n \mathbf{c}_{2}$ is independent of $n \mathbf{c}_{1}$, one must ensure that at least one of $\lambda_{i}^{2} \mathbf{c}_{i}$, $i=2,3, \ldots, w$ is of order $n . \lambda_{1}^{2}$ is not restricted. The number of points available is $n^{w}\left(1-p^{-w+1}\right)$.

Continuing in this way, we find the number of ways of assigning $\mathbf{c}_{k}^{\prime}, k=1,2, \ldots, w$ to be

$$
n^{w}\left(1-p^{-w-1+k}\right) \text {. }
$$

We note again that the result does not depend in detail on the rule form. It depends only on $p$ and on $n_{1}, n_{2}, \ldots, n_{r}$. When several $n_{j}$ are equal, it depends particularly on the pattern. It is notationally convenient to define an integer index.

\section{Definition 4.8 With respect to}

$$
\begin{aligned}
& n_{1} \geq n_{2} \geq n_{3} \geq \ldots \geq n_{r}, \text { the index } \\
& \lambda(j)=k-j
\end{aligned}
$$

where $k$ is the smallest integer for which $n_{k}<n_{j}$.

Theorem 4.9 Given a rule form $Q f$, the number of nontrivial ways of reassigning $\mathbf{c}_{j}$ so that the rule remains unaltered is

$$
\prod_{i=1}^{r} n_{\min (i, j)}\left(1-\frac{1}{p^{\lambda(j)}}\right) .
$$

This number, large as it is, refers only to the number of ways of reassigning $\mathbf{c}_{j}$. Since it is independent of the individual choice for the other generators $\mathbf{c}_{i}(i \neq j)$, we obtain the total number of nontrivial assignments as the product of $r$ corresponding terms. Thus, we have the following corollary.

\section{Corollary 4.10}

$$
\begin{aligned}
\overline{\tilde{\mu}}_{s}\left(n_{1}, n_{2}, \ldots, n_{s}: N\right) & =\prod_{j=1}^{r} n_{j}^{j} \prod_{i=j-1}^{r} n_{i}\left(1-p^{-\lambda(j)}\right) \\
& =V \prod_{t=1}^{r} n_{t}^{2 t-2}\left(1-p^{-\lambda(\ell)}\right) .
\end{aligned}
$$

\section{The Number of Distinct Prime Power Rules having Specified Invariants}

In the previous section we derived a formula for $\overline{\bar{\mu}}_{s}\left(n_{1}, n_{2}, \ldots, n_{s} ; N\right)$. This is the number of distinct assignments for $\mathbf{c}_{1}, \mathbf{c}_{2}, \ldots, \mathbf{c}_{r}$ that give rise to the same rule $Q$. The formula is the same for any rule having these invariants. We may exploit this fact to derive a formula for the number of distinct rules $\nu_{s}\left(n_{1}, n_{2}, \ldots, n_{s} ; N\right)$ having these invariants. 
Definition $5.1 \bar{\mu}_{s}\left(n_{1}, n_{2}, \ldots, n_{s} ; N\right)$ is the number of distinct ways of assigning the $Z$ matrix so that the rule has these invariants. (Here $\mathbf{z}_{k} \in\left[0, n_{k}\right)$.)

Geometrically, this is the number of different point sets $\mathbf{c}_{1} ; \mathbf{c}_{2}, \ldots, \mathbf{c}_{r}$ that can $\mathrm{h}$. substituted into (4.1) to give a nonrepetitive rule form.

Comparing Definition 5.1 with Definition 4.3 shows that $\bar{\mu}_{s}$ is larger than $\overline{\bar{\mu}}_{s}$. Since the redundancy is the same for each distinct rule, it follows that

$$
\nu_{s}\left(n_{1}, n_{2}, \ldots, n_{s} ; N\right)=\frac{\bar{\mu}_{s}\left(n_{1}, n_{2}, \ldots, n_{s} ; N\right)}{\overline{\bar{\mu}}_{s}\left(n_{1}, n_{2}, \ldots, n_{s} ; N\right)} .
$$

The determination of $\bar{\mu}_{s}$ is an exercise of the same type as the determination of $\overline{\bar{\mu}}_{s}$ but much easier in detail. (The formula does not bring in the pattern of $n_{i}$ explicitly.)

The rule may be defined by an assignment of $r$ points $\mathbf{c}_{1}, \mathbf{c}_{2}, \ldots, \mathbf{c}_{r} .\left(\mathbf{c}_{i}=\mathbf{z}_{i} / n_{i}\right.$ is a point of order $n_{i}$ ) $\bar{\mu}_{s}$ is the number of distinct point assignments. This is calculated as $\prod_{j=1}^{r} P_{j}$, where $P_{j}$ is the number of was of choosing $j$ once $\mathbf{c}_{1}, \mathbf{c}_{2}$, and $\mathbf{c}_{j-1}$ have already been assigned.

The number of points of order $n_{j}$ is $n_{j}^{s}\left(1-\frac{1}{p^{s}}\right)$. (This is because when $n=p^{\alpha}$, the grid containing $n^{s}$ points contains all points of orders $1, p, \ldots, p^{\alpha}$. The number of order precisely $p^{\alpha}$ is $\left(p^{\alpha}\right)^{s}-\left(p^{\alpha-1}\right)^{s}$.) However, if $\mathbf{c}_{1}, \mathbf{c}_{2}, \ldots, \mathbf{c}_{j-1}$ have already been assigned, in view of Theorem 3.2, $\mathbf{c}_{j}$ must be chosen so that the order $p$ points it introduces have not been included previously. There are in total $p^{s}-1$ order $p$ points. The number of these in the span of $\mathbf{c}_{1}, \mathbf{c}_{2}, \ldots, \mathbf{c}_{j-1}$ is $p^{j-1}-1$. Thus, a proportion $\left(p^{j-1}-1\right) /\left(p^{s}-1\right)$ of the order $p$ points is not available; alternatively stated, a proportion $\left(p^{s .}-p^{j-1}\right) /\left(p^{s}-1\right)=\left(1-p^{-s+j-1}\right) /\left(1-p^{-s}\right)$ is available. It follows relatively painlessly that of the $n_{j}^{s}\left(1-p^{-s}\right)$ points of order $n_{j}$, only that proportion give rise to available order $p$ points. Thus, $\mathrm{c}_{j}$ may be chosen in $n_{j}^{s}\left(1-p^{j-1-s}\right)$ ways. Hence,

$$
\begin{aligned}
\bar{\mu}_{s} & =\prod_{j=1}^{r} P_{j}=\prod_{j=1}^{r} n_{j}^{s}\left(1-p^{j-1-s}\right) \\
& =N^{s} \prod_{j=1}^{r}\left(1-p^{j-1-s}\right) .
\end{aligned}
$$

The final stage gives the following theorem.

\section{Theorem 5.4}

$$
\nu_{s}\left(n_{1}, n_{2}, \ldots, n_{s} ; N\right)=\prod_{j=1}^{\lfloor s / 2\rfloor}\left(\frac{n_{j}}{n_{s+1-j}}\right)^{s-2 j+1} \prod_{j=1}^{r} \frac{\left(1-1 / p^{s+1-j}\right)}{\left(1-1 / p^{\lambda(j)}\right)} .
$$

Here $n_{1} \geq n_{2} \geq \ldots \geq n_{r}>1$, and we have set $n_{j}=1$ for all $j \in[r+1, s]$. The index $\lambda(j)$ is defined in $(\{, 8)$. 
With a single exception, $v_{s}>1$. The exception is the $s$-dimensional $m$ copy rule. Here all the invariants are equal, and the set $\lambda(j)$ cumprises the first $s$ positive integers.

The first three cases are

$$
\begin{gathered}
\nu_{s}\left(n_{1}, 1^{s-1} ; N\right)=N^{s-1}\left(1-1 / p^{s}\right) /(1-1 / p) \\
\nu_{s}\left(n_{\mathrm{i}}, n_{2}, 1^{s-2} ; N\right)=\left(N^{s-1} / n_{2}^{2}\right)\left(1-1 / p^{s}\right)\left(1-1 / p^{s-1}\right) /\left(1-1 / p^{j:}\right)(1-1 / p),
\end{gathered}
$$

where

$$
\begin{aligned}
j_{1} & =1 \quad \text { when } n_{1}>n_{2}>1 \\
& =2 \text { when } n_{1}=n_{2}>1 \\
\nu_{s}\left(n_{1}, n_{2}, n_{3}, 1^{s-3} ; N\right) & =\frac{N^{s-1}}{n_{2}^{2} n_{3}^{4}} \frac{\left(1-1 / p^{s}\right)\left(1-1 / p^{s-1}\right)\left(1-1 / p^{s-2}\right)}{\left(1-1 / p^{k_{1}}\right)\left(1-1 / p^{k_{2}}\right)(1-1 / p)}
\end{aligned}
$$

where

$$
\begin{array}{lll}
k_{1}=k_{2}=1 & \text { when } & n_{1}>n_{2}>n_{3}>1 \\
k_{1}=3 ; k_{2}=2 & \text { when } & n_{1}=n_{2}=n_{3}>1 \\
k_{1}=2 ; k_{2}=1 & \text { otherwise. } &
\end{array}
$$

These are valid only when $n_{1} \geq n_{2} \geq \ldots \geq n_{r}>1$ and each $n_{i}$ is a power of the same prime $p$.

These results have been obtained independently by Joe and Hunt (1992). Their general approach follows much the same lines as the one here. However, their derivation of $\overline{\bar{\mu}}_{s}$ is embedded in group theory.

These results resemble a similar result (Lyness and Shrevik 1989) for $\nu_{s}(. N)$, the number of distinct lattice rules of order $N$. When $: \mathrm{V}=p^{\beta}$, this is

$$
\nu_{s}(N)=N^{s-1} \prod_{i=1}^{s-1}\left[\left(1-\frac{1}{p^{i+\beta}}\right) /\left(1-\frac{1}{p^{i}}\right)\right] .
$$

\section{A General Formula for $\nu_{s}(\mathbf{n}: N)$}

A simple application of the group decomposition described in Section 2 allows us to base a general formula for $\nu_{s}(\mathrm{n}: . V)$ on the one valid only for prime power rules in Theorem 5.t. The decomposition (2.1) of $G$ into the direct sum of Sylow $p$ gronps has the property that when $\left(i\right.$ has invariants $\mathbf{n}\left(C_{i}\right)$, the invariants $\mathbf{n}\left(S_{p}\right)$ of earh Sylow $p$ group is known. The uniqueness of this decomposition learls to the result that the number of distinct abscissa sets for which $G$ has invariants $\mathbf{n}\left(G^{\prime}\right)$ is the product of the several corresponding numbers for each component $S_{p}$.

Theorem 6.1 Let the prime decomposition of $N$ be

$$
N=p_{1}^{\beta_{1}} p_{2}^{\left(\beta_{2}\right.} \ldots p_{q}^{\beta_{q}} .
$$


Let the invariants $n_{k}$ of a rule $Q$ of order $N$ be given by

$$
n_{k}=p_{1}^{\beta_{1 k}} p_{2}^{\beta_{2 k}} \ldots p_{q}^{\beta_{q k}} \quad k=1,2, \ldots, s .
$$

Then the number of distinct rules $Q$ having these invariants is

$$
\nu_{s}\left(n_{1}, n_{2}, \ldots, n_{s}, N\right)=\prod_{j=1}^{q} \nu_{s}\left(p_{j}^{\beta_{j 1}}, p_{j}^{\beta_{j 2}}, \ldots, p_{j}^{\beta_{j}} ; p_{j}^{\beta_{j}}\right),
$$

where an explicit expression for each of the factors on the right appears in (5.4) above.

In the statement of the above theorem, we have followed various conventions from earlier sections. In particular,

$$
\beta_{j, i} \geq \beta_{j, i+1} .
$$

This theorem is a natural extension of a result in Lyness and Sorevik (1989) to the effect that the number of distinct lattice rules of order $N$ is given by

$$
\nu_{s}(N)=\prod_{j=1}^{q} \nu_{s}\left(p_{j}^{\beta,}\right)
$$

\section{Concluding Remarks}

The general thrust of this article is to provide a straightforward and concrete approach to some of the basic structure of lattice rules. This approach has led through several areas which are new only in a marginal sense.

For example. spelling out the group theory and applying it as in Section 2 is new. Something like it was submitted in an earlier version of Sloan and Lyness (1989) but was excised by the referee. Again, the simple procedure in Section 3 for the reduction of a prime power rule to canonical form has not appeared before. What will soon be available is a more general process in which the Smith Normal Form of a generator marix is used to obtiin a general canonical form. See Lyness and Keast (1991) and Langtry (1991). Howewer, in that process the simple underlying geometry is obscured.

The results of Sections 4 to 6 about the number of lattice rules with given invariants were available to the author in 1989 and promised in 1991. These were subsequently derived independently by Joe and Hunt (1991). In broad outline, their derivation parallels the one given here. However, in their work, $\overline{\tilde{\mu}}_{s}$ appears as the result of a nontrivial argument based on group theory, whereas here it is derived in a direct way as a straightforward redundancy factor in a matrix representation.

This author hopes that this somewhat pedestrian exposition of these ideas will help to enlighten to an elegant branch of numerical quadrature. 


\section{Acknowledgments}

This work was supported in part by the Applied Mathematical Sciences subprogram of the Office of Fnergy Research, U.S. Department of Energy, under Contract W-31109-Eng-38.

\section{References}

Joe S. and Hunt D. C. (1991), "The Number of Lattice Rules Having Given Invariants," Bull. Australian Math. Soc. 46, pp. 477-493. See also Applied Mathematics Preprint AM91/44, UNSW.

Korobov N. M. (1959), "The Approximate Computation of Multiple Integrals" (Russian), Dokl. Akad. Nauk. SSSR 124, pp. 1207-1210.

Lyness J. N. and Keast P. (1991), "Application of the Smith Normal Form to the Structure of Lattice Rules," Preprint MCS-P269-0891, Mathematics and Computer Science Division, Argonne National Laboratory, Aigonne, Ill.

Lyness J. N. and Sфrevik T. (1989), "The Number of Lattice Rules," BIT 29, pp. $527-534$.

Langtry T. N. (1991), "The Determination of Canonical Forms for Lattice Quadrature Rules," private communication.

Niederreiter H. (1992), "Existence Theorems for Efficient Lattice Rules," in Numerical Integration, T. O. Espelid and A. Cenz (eds.), 71-80, Kluwer Academic Publishers, The Netherlauds.

Sloan 1. H. (1992), "Numerical Integraiion in High Dimensions-The Lattice Rule Approach," in Numerical Integration, T. O. Espelid and A. Cienz (eds.), 5.5-69, Kluwer Academic Publishers, The Netherlands.

Sloan I. H. and Lyness J. N. (1989), "The Representation of Lattice Quadrature Rules as Multiple Sums," Math Comput. 52, pp. 81-94.

Dr. J. N. Lyness

Mathematics and Computer Science Division

Argonne National Laboratory

9700 South C'ass Avenue

Argonne, IL 60439

lynessiomes.anl.gov

\section{DISCLAIMER}

This report was prepared as an account of work sponsored by an agency of the United States Government. Neither the United States Government nor any agency thereof, nor any of their cessess or implied, or assumes any legal liability or responsibility for the accuracy, completeness, or usefulness of any information, apparatus, product, or proces the its use would not infringe privately owned rights. Referprocess disclosed, or represents that to any specific commercial product, process, or service by trade name, trademark, ence hecessarily constitute or imply its endorsement, recommendation, or favoring by the United States Government or any agency thereof. The views and opinions of authors expiessed herein do not necessarily state or reflect those of the United States Government or any agency thereof. 

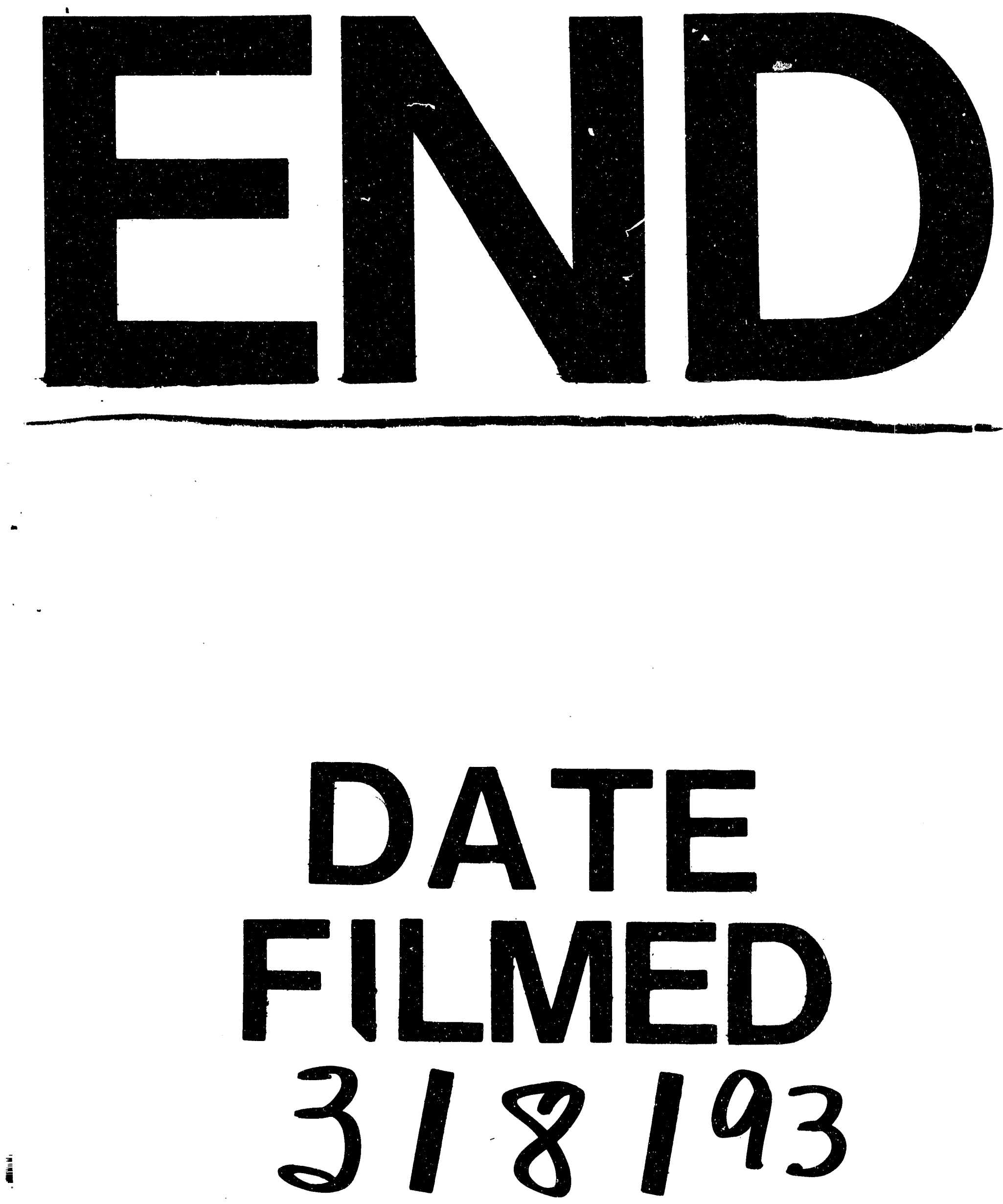
